\title{
La définition d'un espace optimal d'appartenance et de synergies dans un pôle urbain : le cas de Montréal ${ }^{1}$
}

\author{
Peggy Bachman et Jacques Gagnon² \\ Université de Sherbrooke
}

L'environnement dans lequel évoluent les villes aujourd'hui est caractérisé par un certain nombre de tendances marquantes. La mondialisation de l'économie et des politiques constitue sans doute la tendance qui est appelée à se développer, et qui entraîne le plus de changements sur les États tels qu'ils existent aujourd'hui, que ce soit sur leur structure et leur organisation, sur leur économie ou sur leurs caractéristiques sociales. Toutes les orientations auxquelles les États seront confrontés dans les prochaines années seront marquées par le développement et la multiplication des échanges entre les pays, qu'ils soient de nature commerciale, culturelle ou politique.

Dans le système généré par la mondialisation, les bouleversements vécus par les États se répercutent au niveau local, et plus particulièrement sur les pôles urbains, car les contraintes que ce système entraîne pour les États sont intégrées dans les politiques du gouvernement envers les municipalités locales. En effet, plusieurs facteurs sont porteurs de conséquences sur le rôle et la gestion des pôles urbains: il s'agit principalement de la perte de souveraineté des États au profit d'instances internationales ou supranationales, ceci dans plusieurs domaines, notamment en matière de politique économique et monétaire. Les États ne possèdent plus la maîtrise parfaite de leur budget, lequel doit de plus en plus tenir compte de contraintes extérieures. Parallèlement, les États sont également dépendants des flux financiers internationaux, boursiers ou commerciaux (entreprises multinationales). Ces flux imposent aux États des contraintes directes sur leur politique monétaire, et indirectes sur un ensemble d'actions économiques et sociales.

Parallèlement, la perte de souveraineté des États s'accompagne de politiques accrues de décentralisation vers les municipalités locales dans la plupart des pays occidentaux, et particulièrement au Québec et au Canada. Ainsi un grand nombre de services autrefois pris en charge par
l'État est désormais géré par les municipalités locales. Ce phénomène est le fruit d'une longue évolution depuis plusieurs décennies, mais il s'est accéléré depuis 1980 au Québec avec la réforme de la décentralisation, et il est possible de prévoir qu'il s'accentuera encore dans les années à venir. Le transfert massif de compétences et de charges de l'État vers les municipalités locales est notamment une conséquence de la crise des finances publiques nationales, les municipalités locales ayant de cette façon contribué à l'assainissement des finances publiques.

\section{La problématique des grands pôles urbains ou comment assurer une synergie entre compétitivité du territoire et résolution des problèmes sociaux}

La gestion des pôles urbains s'inscrit dans un ensemble de flux entre différents acteurs, dont l'État, mais aussi les autres villes et collectivités locales, les institutions supranationales et internationales, le secteur privé (principalement les entreprises ), le secteur semi-privé ou parapublic ( par exemple les sociétés mixtes), et enfin les communautés locales, représentées par les citoyens et les associations. C'est cet ensemble de flux qui correspond à un système, lequel s'analyse à l'aide d'une approche globale et intégrée de réseau permettant de ne pas se cantonner à une approche limitée par secteur.

\subsection{La complémentarité de la compétitivité et de l'action sociale}

L'analyse intégrée nous conduit à envisager les pôles urbains, pas seulement à la lumière de leurs problèmes internes, mais en lien direct avec leur environnement mondialisé. Le contexte de la mondialisation fait partie intégrante des conditions de développement des pôles urbains: ces derniers doivent composer avec la compétition nationale et internationale face aux autres pôles 
urbains. Chacun tente en effet d'attirer sur son territoire des secteurs entiers de l'économie afin de créer des pôles de développement, au moyen d'incitatifs fiscaux ou d'investissements dans les infrastructures. Dans cet objectif, les institutions municipales urbaines mènent de véritables stratégies de développement, dont les actions s'inscrivent à plusieurs niveaux à la fois : infrastructures, secteur social, éducation, logement, fiscalité, etc.

Dans le cadre de ces fonctions, les grandes villes doivent faire preuve d'une performance économique toujours plus grande. Elles ne doivent pas seulement s'adapter à leur environnement, elles doivent également être capables d'innover afin de se maintenir dans la compétition internationale. Cette capacité d'innovation s'accompagne d'une capacité d'apprentissage, devenue la condition de la performance dans une économie en changement continuel. Ainsi, la performance d'une ville en tant qu'institution et sa capacité d'apprentissage conditionnent la création d'un environnement propice aux développements d'entreprises et l'amélioration de la qualité de vie des citoyens.

\section{La capacité de gérer efficacement ces responsabilités sociales s'intègre dans celle, plus englobante, de devenir et de rester un territoire compétitif et attractif pour les entreprises et pour les ménages. L'attractivité d'un territoire correspond en effet à un ensemble de critères, dont celui de la qualité de vie, qui occupe une place centrale dans les décisions de localisation des entreprises et des ménages. Ainsi le défi majeur des pôles urbains de devenir des pôles attractifs pour les entreprises et pour les ménages repose sur la réalisation d'un équilibre entre les activités économiques et les activités sociales.}

Ce défi est d'autant plus difficile à remplir pour un pôle urbain qu'il est également confronté aux effets pervers de la mondialisation, à savoir l'apparition d'une société à deux vitesses, mettant en jeu des phénomènes sociaux. Afin de s'adapter à ces nouvelles conditions, les municipalités urbaines sont devenues de véritables acteurs du développement local, devant développer des capacités de changement, avec à la fois des responsabilités économiques tournées vers l'international et des responsabilités sociales centrées sur l'espace local. La capacité de gérer efficacement ces responsabilités sociales s'intègre dans celle, plus englobante, de devenir et de rester un territoire compétitif et attractif pour les entreprises et pour les ménages. L'attractivité d'un territoire correspond en effet à un ensemble de critères, dont celui de la qualité de vie, qui occupe une place centrale dans les décisions de localisation des entreprises et des ménages. Ainsi le défi majeur des pôles urbains de devenir des pôles attractifs pour les entreprises et pour les ménages repose sur la réalisation d'un équilibre entre les activités économiques et les activités sociales.

Toutefois, les synergies économiques et sociales au sein de l'espace urbain qui viennent d'être décrites doivent s'accompagner de l'implication des citoyens, dont la condition est l'existence d'un sentiment d'appartenance par rapport à la structure institutionnelle choisie pour gérer les services locaux.

\subsection{La nécessaire implication des citoyens}

Dans le cadre de la mondialisation, le besoin de reconnaissance territoriale prend de l'ampleur en réaction à la crainte de l'uniformisation des économies, des politiques et des cultures. Les structures locales répondent bien à ce besoin de proximité du citoyen. L'identification est cependant plus ardue en milieu urbain qu'en milieu rural, les habitants des milieux urbains étant souvent originaires d'autres régions ainsi que plus mobiles dans l'espace, et par conséquent moins attachés à un territoire unique.

Au-delà du sentiment d'appartenance, l'implication des citoyens exige l'existence de structures démocratiques laissant la place à la consultation, voire à la concertation ou à la participation directe aux prises de décision de l'institution municipale. C'est bien dans la mise en œuvre de ces principes de participation des citoyens que se pose le véritable défi pour les pôles urbains : comment impliquer parfois plusieurs millions de citoyens d'origines et de statuts extrêmement diversifiés ?

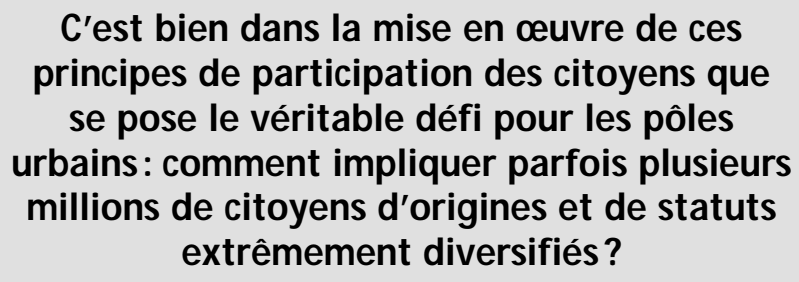

Certains mécanismes existent déjà, au-delà de l'élection de représentants, comme la possibilité pour les munici- 
palités de recourir à des référendums sur des sujets particuliers, ou comme le développement de relations régulières avec des représentants de la société civile (associations de citoyens par exemple). L'implication des habitants des pôles urbains peut être réalisée de multiples manières, à partir du moment où il existe une volonté politique forte dans ce sens. Du côté des populations, l'adhésion sera d'autant plus aisée que l'institution municipale saura démontrer l'efficacité et l'efficience dans sa gestion, alliées à un sentiment d'équité dans la répartition des charges fiscales entre les citoyens.

Dans ce contexte, un espace optimal d'appartenance et de synergies dans un pôle urbain correspondrait à un espace alliant efficience de la gestion des services et équité fiscale.

\subsection{Une structure de gestion optimale}

Il est reconnu que la fragmentation des structures municipales au sein d'une agglomération est un facteur de dysfonctionnement de ces agglomérations. De surcroît, l'ancienne opposition entre centre et périphérie n'est plus de mise dans le contexte de la mondialisation, qui appelle au contraire une pensée globale basée sur la complémentarité des espaces. De nombreux services doivent désormais être pensés et gérés pour l'ensemble d'une agglomération, tant les échanges se multiplient au sein de cette agglomération. Les politiques concernant le transport, mais aussi la culture, l'éducation et le tourisme doivent être conçues à partir des interactions entre les différents espaces. Le développement économique à long terme évolue de manière inégale d'un espace à un autre de l'agglomération. Seule une approche en terme de système permet d'envisager un développement économique profitable à l'ensemble de l'agglomération, et non seulement à quelques territoires privilégiés au détriment de tous les autres.

L'ancienne opposition entre centre et périphérie n'est plus de mise dans le contexte de la mondialisation, qui appelle au contraire une pensée globale basée sur la complémentarité des espaces. De nombreux services doivent désormais être pensés et gérés pour l'ensemble d'une agglomération, tant les échanges se multiplient au sein de cette agglomération.
Il s'agit finalement de substituer une logique de coopération à une logique de concurrence à l'intérieur même d'une agglomération, en vue de la rendre compétitive face à l'extérieur.

\section{La nouvelle ville de M ontréal, un espace optimal d'appartenance et de synergies?}

Rappelons-le, Montréal est la plus grande ville du Québec en termes démographique et économique. Il est donc naturel que lui soit portée une attention particulière de la part du gouvernement du Québec. Désignée sous le terme de métropole, Montréal relève en effet d'un ministère provincial spécifique: le Ministère des Affaires Municipales et de la Métropole.

Le projet de faire de l'île de Montréal une seule ville n'est pas nouveau (il date du début du $\mathrm{XX}^{\mathrm{e}}$ siècle) et, n'ayant jamais été mis en œuvre, il a été relancé ces dernières années. Le projet se voulait d'autant plus urgent que récemment d'autres grandes villes canadiennes avaient fait l'objet de regroupements. Ainsi le gouvernement de l'Ontario a imposé la fusion de la municipalité régionale de Toronto avec ses six municipalités constituantes, qui a pris effet le $1^{\text {er }}$ janvier 1998. Plus généralement, les réformes des structures de gestion municipale sont nombreuses dans le monde, que ce soit en Amérique du Nord ou en Europe.

Montréal ne pouvait conserver ses structures obsolètes, la ville devait s'adapter et changer pour relever le défi de la mondialisation. Le gouvernement du Québec a ainsi effectué un choix, qui a abouti en 2000 à la mise en œuvre d'un processus de regroupement des villes de l'île de Montréal, faisant d'une île une ville.

Selon les recommandations du mandataire du gouvernement concernant les regroupements municipaux dans la région métropolitaine de Montréal ( 11 octobre 2000), le processus de regroupement repose sur l'analyse de l'agglomération de Montréal comme agglomération polycentrique où coexistent trois grands pôles de développement:

- un pôle central, constitué de l'île de Montréal,

- un pôle nord formé de la ville de Laval et de la couronne nord,

- un pôle sud formé de l'ensemble de la couronne sud.

Ce processus se décompose en plusieurs procédures de regroupements, dont celui des municipalités de l'île de 
Montréal (le pôle central), qui ne représente désormais qu'une seule ville (depuis le $1^{\text {er }}$ janvier 2002).

Concernant plus précisément l'aspect de la gestion, le processus de regroupement ayant été mis en œuvre dans l'agglomération de Montréal repose sur trois éléments de gestion de base:

\subsection{Une stratégie de gestion qui vise l'optimisation des ressources sur le territoire}

Ce thème a fait l'objet de nombreux débats à l'occasion de la réforme, et nous ne reviendrons pas sur tous les arguments évoqués de part et d'autres. Nous souhaitons simplement souligner ici que la fragmentation géographique et institutionnelle qui existait avant le regroupement sur l'île de Montréal ne semblait pas propice à une optimisation des ressources humaines, financières et naturelles, puisque chaque municipalité conservait la maîtrise de l'utilisation et de la gestion de ces ressources, sans véritable concertation avec les autres municipalités, et parfois même dans une optique concurrentielle.

La fragmentation géographique et institutionnelle qui existait avant le regroupement sur l'île de Montréal ne semblait pas propice à une optimisation des ressources humaines, financières et naturelles, puisque chaque municipalité conservait la maîtrise de l'utilisation et de la gestion de ces ressources, sans véritable concertation avec les autres municipalités, et parfois même dans une optique concurrentielle.

Il a été évoqué que certaines des anciennes municipalités de la nouvelle ville étaient caractérisées par une excellente gestion, contrairement à l'ancienne ville de Montréal, que ces mêmes municipalités percevaient comme mal gérée. La crainte de la perte de cette bonne gestion au sein de la nouvelle ville a constitué l'une des raisons de l'opposition de certaines municipalités au regroupement. Sur ce point, il est important de rappeler que la nouvelle ville de Montréal, comme son nom l'indique, ne correspond pas à une annexion de municipalités par une autre, mais bien à un véritable regroupement des anciennes municipalités au sein d'une nouvelle. Les élections ont permis de constituer un nouveau conseil municipal. La nouvelle ville et ses représentants nouvellement élus sont désormais libres d'adopter le style de gestion qu'ils souhaitent, qu'il soit totalement nouveau (mais rien n'est jamais totalement nouveau en matière de gestion ) ou qu'il soit emprunté à l'une ou l'autre des anciennes municipalités. Ils ont d'ailleurs le mandat d'améliorer continuellement la gestion de la nouvelle ville. Pour terminer sur ce point, il est utile de préciser que l'objectif ultime de la nouvelle ville est de gérer ses ressources de manière optimale sur l'ensemble d'un territoire qui représente aujourd'hui une réalité et une cohérence économiques et sociales, bref un bassin de vie.

\subsection{Une stratégie de développement économique}

La stratégie de développement économique inscrite dans le processus de regroupement de Montréal consiste à créer des pôles de développement aptes à répondre aux besoins et aux exigences de développement à la fois régionaux, canadiens et internationaux.

Montréal constitue la métropole du Québec, elle est aussi l'une des plus importantes métropoles du Canada, enfin elle est une métropole internationale ${ }^{3}$. Il s'agit là d'un constat, qui est révélateur de l'enjeu de développement que Montréal représente pour le Québec. Dans le contexte de concurrence qui a été décrit plus haut, Montréal doit poursuivre une stratégie de développement visant à en faire une métropole compétitive face aux autres métropoles canadiennes et nord-américaines, voire sur la scène internationale. Dans cet objectif de compétitivité face à l'extérieur, il était impératif de doter Montréal d'une structure de gestion cohérente avec son territoire. Les anciennes limites de Montréal ne représentaient plus la réalité de la ville ni sa perception par l'extérieur. De surcroît, ces limites handicapaient son développement économique, qui présentait un défaut de cohérence et de synergie.

\subsection{Une gestion du territoire cohérente}

La recherche d'une gestion cohérente du territoire de Montréal consiste à tenir compte des besoins du milieu tout en assurant un développement durable qui permet une équité fiscale et une régulation de la problématique de l'étalement urbain.

Un aspect important de l'utilité de la réforme pour Montréal correspond à la nécessité en terme d'équité de rapprocher les coûts des services de leurs utilisateurs, et d'établir une certaine solidarité entre les différentes couches de la population. L'application, à terme, de taux 
uniformisés de taxation sur l'ensemble du territoire représente un instrument important pour atteindre ces objectifs. De plus, cette uniformisation devrait permettre de limiter les mouvements favorisant l'étalement urbain, bien qu'elle ne suffira pas à elle-seule à résoudre ce problème.

\section{Un aspect important de l'utilité de la réforme pour Montréal correspond à la nécessité en terme d'équité de rapprocher les coûts des services de leurs utilisateurs, et d'établir une certaine solidarité entre les différentes couches de la population. L'application, à terme, de taux uniformisés de taxation sur l'ensemble du territoire représente un instrument important pour atteindre ces objectifs.}

Les métropoles du monde entier sont confrontées à ces problèmes. Les solutions adoptées ne sont pas toutes semblables, mais elles passent généralement par un objectif d'uniformisation des taux de taxation locale. Pour emprunter un exemple parfois méconnu, les solutions adoptées en France illustrent bien cet objectif: de récentes réformes visent à mettre en œuvre un taux unique de taxe professionnelle 4 à l'échelle des agglomérations, qui sont gérées par des communautés urbaines ou par des communautés d'agglomération possédant un pouvoir de taxation.
Ainsi, la création de la nouvelle ville de Montréal devrait se rapprocher des conditions de réalisation d'un espace optimal d'appartenance et de synergies. Pour que cette réalisation soit effective, il faudra prêter une attention toute particulière à la nouvelle Communauté métropolitaine de Montréal, dont le rôle sera de finaliser la cohérence territoriale amorcée avec les regroupements municipaux. Il ne faut pas oublier que le bassin de vie que représente l'île de Montréal s'insère dans un système beaucoup plus large qui s'étend jusqu'aux rives Sud et Nord de la métropole.

\section{Notes et références}

1 Ce texte a fait l'objet d'une présentation avec deux autres textes des auteurs lors des Entretiens Jacques Cartier à Lyon en décembre 2001.

2 Peggy Bachman est doctorante à l'Université de Sherbrooke et l'Université Jean Moulin Lyon III en France. Elle est conseillère en fiscalité à l'Union des municipalités du Québec. Jacques Gagnon est professeur au département de management de l'Université de Sherbrooke et titulaire de la Chaire J.W. McConnell en développement local. Il est également membre du Centre d'excellence en gestion du développement local.

3 Nous faisons référence ici à la définition d'une ville internationale proposée par P. Soldatos (1991). Les nouvelles villes internationales: profil et planification stratégique. Aix-enProvence: Serdeco.

4 La taxe professionnelle s'applique uniquement aux activités économiques, mais elle représente la moitié des recettes fiscales locales en produit. 